Sharif University of Technology
Scientia Iranica
Transactions E: Industrial Engineering
hCIttp://scientiairanica.sharif.edu
IRAN I CA

\title{
Statistical adjustment and calibration of complex systems considering multiple outputs: Case study of laser-assisted micromachining process
}

\author{
Z. Khalaj, A. Aghaie*, and Y. Samimi \\ Department of Industrial Engineering, K.N. Toosi University, Tehran, 1999143344, Iran.
}

Received 24 June 2019; received in revised form 10 August 2020; accepted 28 December 2020

KEYWORDS
Uncertainty
quantification;
Adjustment;
Calibration;
Engineering-statistical
model;
Gaussian process;
Laser-assisted
micromachining
process.

\section{Introduction}

In the real world, we are faced with uncertainty in everyday life. In simulating complicated systems, making management decisions, evaluating the performance of systems in different situations, and then optimizing them, the uncertainty plays a critical role. To better

\footnotetext{
*. Corresponding author.

E-mail addresses: zkhalaj@mail.kntu.ac.ir (Z. Khalaj); aaghaie@kntu.ac.ir (A. Aghaie); Y_samimi@kntu.ac.ir (Y. Samimi)
}

doi: $10.24200 /$ sci.2020.53857.3455

\begin{abstract}
With rapid advancements in technologies, studying and simulating a real complex system taint with uncertain parameters has become extremely demanding. Based on the relevant literature, there are three approaches to recognizing and simulating different systems, namely engineering, statistical, and engineering-statistical. Regarding the purpose of this research, by considering two outputs, simultaneously, Laser Assisted Micro-Machining (LAMM) was studied by adopting the engineering-statistical approach. Investigating variates simultaneously pose some complicated issues, such as calibrating variates at the same time, adjusting them concurrently, and calculating the values of parameters, with which this paper should cope. Considering Mean Squared Prediction Error (MSPE) as the comparison index for the thrust force output, the index value was obtained 1.48 by the Kennedy and O'Hagan model, 2.47 by the model presented by Roshan and Yan, and $1.9425 \times 10^{-4}$ by the proposed model. Moreover, for the cutting force output, the index was obtained 0.21 by the Kennedy and O'Hagan, 1.41 by Roshan and Yan, and $1.6 \times 10^{-8}$ by the presented model. The obtained values demonstrated reasonable and acceptable results for the MSPE index compared with the models that considered the outputs individually.
\end{abstract}

(C) 2022 Sharif University of Technology. All rights reserved. cope with the uncertainty, different models can be defined. A model is a representation of a system, person, thing, or a given structure, typically on a smaller scale than the original one. According to recent studies, three approaches are generally adopted to model real systems, including engineering, statistical, and engineering-statistical. These approaches are described as follows.

The first approach to model a system is called the engineering approach. Engineering models, which are developed by applying some techniques such as finite element and numerical analysis, are considered physicsbased and fulfill the physical interpretation of a system. Accordingly, they considerably help understand the 
real system. However, to accomplish this approach, some simplifying assumptions are considered, which make the models almost unrealistic. Furthermore, it is a time-consuming procedure to search for the reasons of discrepancy between a developed model and the observed data in order to fix all the wrong assumptions. Therefore, if the developed model does not operate properly, it takes a long time to find the error and change it and then, reformulate the model. In the following, some of the studies, which have recently been conducted in this field, are presented in Table 1. In this field, Singh and Melkote's research [1] is one of the most striking papers.

The second approach is the statistical one by which an empirical model (be a GP or a regression model) is built to capture the discrepancies. In this approach, real data are collected from the systems to estimate the unknown parameters of the model (calibration parameters). The first disadvantage of this approach is that any changes in the system result in repeating the data collection procedure, developing a new model, and collaborating it again. Moreover, the approach does not respond well out of experimental conditions and it lacks physical interpretation. In this regard, some relevant research is reported in Table 1. Among the conducted research, Kennedy and O'Hagan [2] and Roshan and Melkote [3] are the most influential ones on the current study. It is noteworthy that statistical inference, Bayesian Approach (BA), Gaussian Process (GP), Maximum Likelihood Estimation (MLE), response surface methodology, Analysis of Variance (ANOVA), regression, and robust techniques are the most popular approaches in this field.

By the integration of the two previous approaches, the third approach, namely the engineering-statistical approach, is developed. This approach begins with building and calibrating an engineering model (by collecting data), which fulfills the physical interpretation, and then, distinguishes the discrepancy and its causes using ANOVA; finally, it tries to eliminate the discrepancy by applying statistics. At the next step, the adjustment models are postulated and eventually, the final model fulfills a physical interpretation. Contrary to the two previous approaches, this approach is not time-consuming, which is considered as its distinctive feature. In this respect, relevant works done on this approach are presented in Table 1. For more information, the interested readers are referred to Yan [4], Roshan and Yan [5], and Sheikhi and Saghaie [6] which apply the Computational Fluid Dynamics (CFD), BA, and GP respectively.

It should be noted in the real world, some of the systems are too complicated to be easily modeled. In these situations, engineering approaches, including the finite element and CFD, are applied to modeling the system. These models may be formed by the combination of multiple models, each of which presents a specific part of the system. For instance, such systems have more than one output variable, mostly; hence, for each variable, a different model can be used. Needless to say, these models are not concise and there is some bias between the model values and real observations. In order to cope with the problem, statistical approaches are applied for calibrating and adjusting such complicated models, which leads to the engineering-statistical approach.

\subsection{Contribution of the present study}

A summary of studies regarding each system modeling approach is demonstrated in Table 1. Subsequently, in this paper, an engineering-statistical approach is adopted to fulfill the drawbacks of both the statistical and engineering approaches. It is clear that although the previously proposed engineering-statistical procedures have been of single-output type $[4,6,7]$, studying the covariance and correlation between the outputs may end up in more appropriate models to get better results. Therefore, instead of applying BA, which needs prior distribution as well as hard usage for multiple outputs, MLE and GP are applied.

\section{Methodology}

This section is aimed at providing the proposed approach. As a basic model, Kennedy and O'Hagan [2] presented Model (1) for a system. In this model, $y$ implies the output of the system, $x=\left(x_{1} \ldots x_{p}\right)$ shows the vector of input variables, and $f(x ; \eta)$ plays the role of an engineering model. Moreover, $\eta=\left(\eta_{1} \ldots \eta_{p}\right)$ reflects the vector of calibration parameters of the engineering model:

$$
y=\rho f(x ; \eta)+\delta(x)+\varepsilon .
$$

where, $\rho$ is the scale parameter, $\delta(x)$ denotes the discrepancy function (model bias), and the random error is presented by $\varepsilon_{\sim}^{i i d} N\left(0, \sigma^{2}\right)$. Then, by considering $h(x)=\left(h_{o}(x) \ldots h_{l}(x)\right)$ as a set of known functions and $\mu=\left(\mu_{o} \ldots \mu_{l}\right)$, Kennedy and O'Hagan defined a GP for the discrepancy:

$$
\delta(x) \sim G P\left(h(x)^{\prime} \mu, \tau^{2} R(.)\right) .
$$

The covariance function is presented by:

$$
\operatorname{cov}\left(\delta\left(x_{i}\right), \delta\left(x_{j}\right)\right)=\tau^{2} R\left(x_{i}-x_{j}\right),
$$

in which $\tau^{2}$ is the variance of $\delta(x)$ and $R$ is an $n \times n$ correlation matrix.

Inspired by Kennedy and O'Hagan's paper, Roshan and Yan [5] considered $\rho=1$ and $\mu=0$; thus, Models (1) and (2) were changed to Model (3):

$$
\begin{aligned}
& y=f(x ; \eta)+\delta(x)+\varepsilon, \\
& \delta(x) \sim G P\left(0, \tau^{2} R(.)\right) .
\end{aligned}
$$


Table 1. A summary of studies considering each approach as well as the applied methods.

\begin{tabular}{|c|c|c|c|c|c|c|c|c|c|c|c|c|c|c|c|}
\hline \multirow[b]{2}{*}{ Authors } & \multicolumn{3}{|c|}{ Approach } & \multicolumn{12}{|c|}{ Methods } \\
\hline & 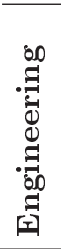 & 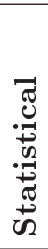 & 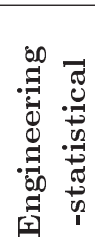 & 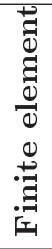 & 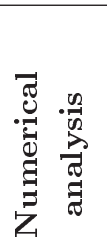 & 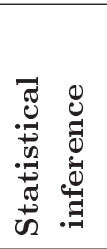 & 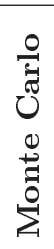 & 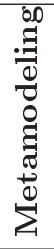 & $\underset{m}{\sharp}$ & O) & $\begin{array}{l}4 \\
3 \\
0 \\
Z\end{array}$ & 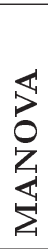 & 国 & 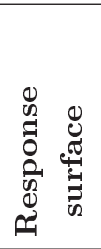 & 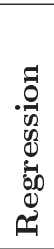 \\
\hline Allaire [11] & $\bullet$ & - & - & & $\bullet$ & - & - & - & - & - & - & - & - & - & - \\
\hline Nocedal and Wright [12] & $\bullet$ & - & - & $\bullet$ & - & - & - & - & - & - & - & - & - & - & - \\
\hline Szabó [13] & $\bullet$ & - & - & $\bullet$ & - & - & - & - & - & - & - & - & - & - & - \\
\hline Mathelin and Hussaini [14] & • & - & - & $\bullet$ & - & - & - & - & - & - & - & - & - & - & - \\
\hline Singh and Melkote [1] & $\bullet$ & - & - & $\bullet$ & - & - & - & - & - & - & - & - & - & - & - \\
\hline Vepsä et al. [15] & $\bullet$ & - & - & $\bullet$ & - & - & - & - & - & - & - & - & - & - & - \\
\hline Palumbo et al. [16] & $\bullet$ & - & - & $\bullet$ & - & - & - & - & - & - & - & - & - & - & - \\
\hline Stickler and Schachinger [17] & $\bullet$ & - & - & $\bullet$ & - & - & - & - & - & - & - & - & - & - & - \\
\hline Sena and Silvapulle [18] & - & $\bullet$ & - & - & - & $\bullet$ & - & - & - & - & - & - & - & - & - \\
\hline Kumar et al. [19] & - & $\bullet$ & - & - & - & $\bullet$ & - & - & - & - & - & - & - & - & - \\
\hline Roussas [20] & - & $\bullet$ & - & - & - & $\bullet$ & - & - & - & - & - & - & - & - & - \\
\hline Zio [21] & - & $\bullet$ & - & - & - & - & $\bullet$ & - & - & - & - & - & - & - & - \\
\hline Dong et al. [22] & - & $\bullet$ & - & - & - & - & $\bullet$ & - & - & - & - & - & - & - & - \\
\hline Parnianifard et al. [23] & - & $\bullet$ & - & - & - & - & - & $\bullet$ & - & - & - & - & - & - & - \\
\hline Kennedy and O'Hagan [2] & - & $\bullet$ & - & - & - & - & - & $\bullet$ & $\bullet$ & $\bullet$ & $\bullet$ & - & - & - & - \\
\hline Roshan and Melkote [3] & - & $\bullet$ & - & - & - & - & - & $\bullet$ & $\bullet$ & $\bullet$ & $\bullet$ & - & - & - & $\bullet$ \\
\hline Park and Grandhi [24] & - & $\bullet$ & - & - & - & - & - & - & $\bullet$ & - & - & - & - & - & - \\
\hline Caiado and Goldstein [25] & - & $\bullet$ & - & - & - & - & - & - & $\bullet$ & - & - & - & - & - & - \\
\hline Wong et al. [26] & - & $\bullet$ & - & - & - & - & - & - & $\bullet$ & - & - & - & - & - & - \\
\hline Sankararaman and Mahadevan [27] & - & $\bullet$ & - & - & - & - & - & - & $\bullet$ & - & - & - & - & - & - \\
\hline Duru et al. [28] & - & $\bullet$ & - & - & - & - & $\bullet$ & - & $\bullet$ & $\bullet$ & - & - & - & - & - \\
\hline Azzimonti et al. [29] & - & $\bullet$ & - & - & - & - & - & - & $\bullet$ & $\bullet$ & - & - & $\bullet$ & - & - \\
\hline Reid [30] & - & $\bullet$ & - & - & - & - & - & - & - & - & - & - & $\bullet$ & - & - \\
\hline Junaid and Wani [31] & - & $\bullet$ & - & - & - & - & - & - & - & - & $\bullet$ & - & - & $\bullet$ & - \\
\hline Brandt [32] & - & $\bullet$ & - & - & - & - & $\bullet$ & - & - & - & - & - & $\bullet$ & - & - \\
\hline Korunović et al. [33] & - & $\bullet$ & - & - & - & - & - & - & - & - & $\bullet$ & - & - & - & $\bullet$ \\
\hline Lin and Lin [34] & - & $\bullet$ & - & - & - & $\bullet$ & - & - & - & - & - & - & - & - & - \\
\hline Lunardon and Ronchetti [35] & - & $\bullet$ & - & - & - & - & - & - & $\bullet$ & - & - & - & - & - & - \\
\hline Pratola and Higdon [36] & - & $\bullet$ & - & - & - & - & - & - & $\bullet$ & - & - & - & - & - & - \\
\hline Recep et al. [37] & - & $\bullet$ & - & - & - & - & - & - & $\bullet$ & - & - & - & - & - & - \\
\hline Saikumar et al. [38] & - & $\bullet$ & - & - & - & - & - & - & $\bullet$ & - & - & - & - & - & - \\
\hline Chen et al. [39] & - & $\bullet$ & - & - & - & - & - & - & $\bullet$ & - & - & - & - & - & - \\
\hline Neal [40] & - & $\bullet$ & - & - & - & - & - & - & & $\bullet$ & - & - & - & - & - \\
\hline Mondal et al. [41] & - & $\bullet$ & - & - & - & - & - & - & $\bullet$ & - & - & - & - & - & - \\
\hline Díaz-García, [42] & - & $\bullet$ & - & - & - & $\bullet$ & - & - & & - & - & $\bullet$ & - & - & - \\
\hline Rahimi et al. [7] & - & $\bullet$ & - & - & - & - & - & $\bullet$ & $\bullet$ & - & - & - & - & - & - \\
\hline Yan [4] & - & - & $\bullet$ & $\bullet$ & - & - & - & $\bullet$ & $\bullet$ & $\bullet$ & $\bullet$ & - & - & $\bullet$ & - \\
\hline Roshan and Yan [5] & - & - & $\bullet$ & $\bullet$ & - & - & - & $\bullet$ & $\bullet$ & $\bullet$ & $\bullet$ & - & - & - & $\bullet$ \\
\hline Yan et al. [9] & - & - & $\bullet$ & $\bullet$ & - & - & - & $\bullet$ & $\bullet$ & $\bullet$ & $\bullet$ & - & - & - & $\bullet$ \\
\hline Sheikhi and Saghaie [6] & - & - & $\bullet$ & - & - & - & - & - & $\bullet$ & $\bullet$ & $\bullet$ & - & - & $\bullet$ & - \\
\hline Singh et al. [10] & - & - & $\bullet$ & - & - & - & - & $\bullet$ & $\bullet$ & $\bullet$ & $\bullet$ & - & - & - & $\bullet$ \\
\hline Current study & - & - & $\bullet$ & - & - & - & - & $\bullet$ & $\bullet$ & $\bullet$ & - & $\bullet$ & $\bullet$ & - & $\bullet$ \\
\hline
\end{tabular}


As the first step of their algorithm, the model discrepancy was estimated and its causes were detected. Next, the Gaussian correlation function could be defined as:

$$
R\left(x_{i}-x_{j}\right)=\exp \left\{-\sum_{k=1}^{p} \theta_{k}\left(x_{i k}-x_{j k}\right)^{2}\right\} .
$$

Therefore, $\eta$ and $\phi=\left(\sigma^{2}, \tau^{2}, \theta^{\prime}\right)$ remain as unknown parameters. Then, to estimate the values of $\eta$ and $\phi$, $\delta(x)$ is integrated out from the joint posterior, resulting in:

$$
\begin{aligned}
& p(\eta, \phi \mid y) \propto \frac{1}{\left|\tau^{2} R+\sigma^{2} I\right|^{1 / 2}} \\
& \quad \exp \left\{-\frac{1}{2} \frac{(y-f(\eta))^{\prime}}{\tau^{2} R+\sigma^{2} I}(y-f(\eta))\right\} p(\eta, \phi),
\end{aligned}
$$

in which $R$ is a $n \times n$ correlation matrix $(R(i, j)=$ $\left.R\left(x_{i}-x_{j}\right)\right) ; I$ is the identity matrix $(n \times n) ; f(\eta)=$ $\left(f\left(x_{1} ; \eta\right), \ldots, f\left(x_{n} ; \eta\right)\right)$; and $y=\left(y_{1}, \ldots, y_{n}\right)$. Subsequently, considering $r(x)=\left(R\left(x-x_{1}\right), \ldots, R\left(x-x_{n}\right)\right)$, the discrepancy function is given by:

$$
\delta(x) \sim G P\left(h(x)^{\prime} \mu, \tau^{2} R(.)\right) .
$$

Roshan and Yan [5] showed that the prediction variance would be calculated by Eq. (7):

$$
s^{2}(x)=\sigma_{f}^{2}+\sigma_{f}^{2} \dot{f}(x)^{\prime}\left(\dot{F}^{\prime} \dot{F}\right)^{-1} \dot{f}(x)
$$

in which $\dot{F}=\left(\dot{f}\left(x_{1}\right), \ldots, \dot{f}\left(x_{n}\right)\right)$ and $\dot{f}(x)$ is the gradient function of $\hat{f}(x)$ according to $x$ :

$$
\dot{f}(x)=\hat{f}(x)\left(\frac{\hat{\beta}_{1}}{x_{1}}, \hat{\beta}_{2},-\hat{\beta}_{3} e^{-\hat{\beta}_{4} x_{4}}, \hat{\beta}_{3} \hat{\beta}_{4} x_{3} e^{-\hat{\beta}_{4} x_{4}}\right) .
$$

Next, applying ANOVA, the main effects of the engineering model and the discrepancy function are studied. Then, a prior inverse-Gamma is specified for $\sigma^{2}$. In the next step, calculating the prediction variance, the parameters are estimated. By identifying the factors with significant effects on the model discrepancy, the scale adjustment model will be obtained by Eq. (9). A variety of common methods for the calibration of parameters in multiple correlated responses are classified by [8].

$$
g(x ; \eta, \gamma)=f\left(\eta_{1} x_{1}, \ldots, \eta_{p} x_{p} ; \gamma\right) .
$$

It should be noted the above methodology studies only single-output models. In this manuscript, a methodology is proposed to study multivariate systems. Herein, all of the outputs are considered simultaneously.

In the following, the proposed model and methodology are studied. For $q$ variables, the model is changed to:

$$
\left\{\begin{array}{l}
y_{i}=f_{i}(x ; \eta)+\delta_{i}(x)+\varepsilon_{i} ; i=1, \ldots, q \\
\delta_{i}(x) \sim G P\left(0, \tau_{i}^{2} R_{i}(.)\right) ; i=1, \ldots, q \\
\left(\varepsilon_{1}, \ldots, \varepsilon_{q}\right) \sim M V N\left(0,\left(\sigma_{1}^{2}, \ldots, \sigma_{q}^{2}\right)\right) .
\end{array}\right.
$$

Optimizing $f_{i}(x ; \eta)$, the calibration parameters $\eta_{i}$ are obtained. In this respect, an iterative algorithm is used, which updates the error covariance matrix in every iteration. That is, in every single step, the covariance matrix is replaced with a new one. This dynamic approach helps improve the solution and bring it closer to its exact value. To optimize $f_{i}(x ; \eta)$, the MLE method is used. Then, Gaussian processes will be fitted for $\delta_{i}(x)$ using the squared exponential kernel function with default kernel parameters. Predicting $\delta_{i}(x)$ for each run, errors are estimated as:

$$
\varepsilon_{i}=y_{i}-\left(f_{i}\left(x ; \eta_{i}\right)+\delta_{i}(x)\right) ; \quad i=1, \ldots, q .
$$

The bias-corrected engineering model is given by:

$$
\hat{y}_{i}=f_{i}\left(x ; \eta_{i}\right)+\hat{\delta}_{i}(x) .
$$

Now, Multivariate Analysis of Variance (MANOVA) decomposition is performed on $q$ variables, simultaneously, and the significant factors of $f_{i}$ can be easily obtained. $f_{i}\left(x_{i}\right)$ and $\hat{y}_{i}\left(x_{i}\right)$ can be plotted side by side to understand the changes in main effects due to discrepancies and to look at the main effect plots, making the procedure simpler. In order to detect the factors with significant effects on the model discrepancy, the scale adjustment model will be given by Eq. (9). The procedure is stated in Figure 1.

By following the above procedure, the engineering model and the real data are achieved and the model is calibrated by MLE estimation. Calculating the errors and applying the iterative algorithm, the covariance matrix is updated until the calibrated model is adequate. Then, the discrepancy model is calculated by a GP and its causes are detected by MANOVA. Calculating the errors and updating the covariance matrix, the whole procedure will be repeated to reach adequacy. The parameters and variables are stated in Table 2.

The methodology is applied to Laser-Assisted Micro-Machining (LAMM), studied by $[1,7,9]$. In this problem, four variables and two outputs are considered. Roshan and Yan [5] worked on cutting force as one of the outputs. In the present study, thrust force, as another output, is studied simultaneously as presented in Section 3.

\section{Numerical example}

A case study of LAMM was studied previously by some researchers. However, an engineering model was developed by Singh and Melkote [1], calibrated by Singh et al. [10], and adjusted statistically by Roshan and Yan [5]. Roshan and Yan [5] stated that the 


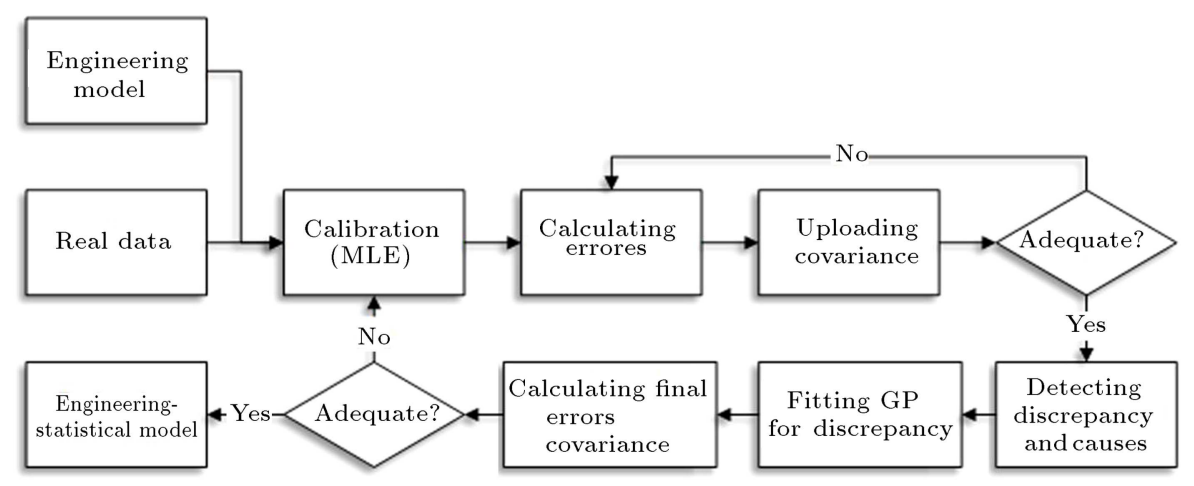

Figure 1. The proposed procedure

Table 2. Description of parameters and variables.

\begin{tabular}{cl}
\hline Parameter/variable & \\
\hline$Y=\left(y_{1} \ldots y_{q}\right)$ & The output variables \\
$q$ & Number of the output variables \\
$p$ & The vector of input variables \\
$\eta=\left(x_{1} \ldots x_{p}\right)$ & Number of the input variables \\
$f(x ; \eta)$ & The vector of calibration parameters of the engineering model \\
$\rho$ & The engineering model \\
$\delta(x)$ & The scale parameter \\
$\varepsilon$ & The discrepancy function (model bias) \\
$\sigma^{2}$ & The random error \\
$\mu=\left(\mu_{0} \ldots \mu_{l}\right)$ & The mean of the discrepancy function \\
$\tau^{2}$ & The variance of the discrepancy function \\
$R$ & $n \times n$ correlation matrix \\
$\theta$ & The characteristic length scale \\
$\gamma$ & The vector of adjustment parameters of the engineering-statistical model \\
\hline
\end{tabular}

Table 3. Levels of variables based on the model presented by Singh et al. [10].

\begin{tabular}{ccccc}
\hline Variable & \multicolumn{4}{c}{ Levels } \\
\hline$X_{1}$ & 10 & 15 & 20 & 25 \\
$X_{2}$ & 10 & 50 & & \\
$X_{3}$ & 0 & 5 & 10 & \\
$X_{4}$ & 100 & 200 & & \\
\hline
\end{tabular}

engineering model consisted of a geometric model for computing strain rates, a finite element model for computing temperature distribution, a material model for computing stresses, a force model for computing forces, and an iterative algorithm to account for the machine-tool-workpiece deflection.

In Table 3, four variables are considered: nominal depth of cut $\left(x_{1}\right)$, speed $\left(x_{2}\right)$, laser power $\left(x_{3}\right)$, and laser location $\left(x_{4}\right)$ at $4,2,3$, and 2 levels, respectively. Two outputs, namely cutting force and thrust force, have been studied separately. However, in this paper, they are studied simultaneously. For these two variables, the model is presented by model Eq. (13):

$$
\left\{\begin{array}{l}
y_{i}=f_{i}(x ; \eta)+\delta_{i}(x)+\varepsilon_{i} ; \quad i=1,2 \\
\delta_{i}(x) \sim G P\left(0, \tau_{i}^{2} R_{i}(.)\right) ; \quad i=1,2 \\
\left(\varepsilon_{1}, \varepsilon_{2}\right) \sim \operatorname{MVN}\left(0,\left(\sigma_{1}^{2}, \sigma_{2}^{2}\right)\right) .
\end{array}\right.
$$

Since the engineering models are complicated and time-consuming (14 hours) besides being expensive to evaluate [5], $f(x ; \eta)$ is replaced by a metamodel, which is considered easy-to-evaluate. The metamodel used by Singh et al. [10] for both output variables is shown below:

$$
f_{i}(x)=b_{0} x_{1}^{b_{1}} \exp \left\{b_{2} x_{2}-b_{3} x_{3} \exp \left(-b_{4} x_{4}\right)\right\} .
$$

As the above model is nonlinear, nonlinear regression is applied for both $f_{1}$ and $f_{2}$ to achieve the parameters $b_{0}, \ldots, b_{4}$. The covariance between two forces is considered as the initial covariance and is updated 
frequently to achieve the optimal solution. Optimizing the engineering model, using MLE and the iterative algorithm, and considering the covariance between the outputs $\left(\operatorname{cov}\left(\delta\left(x_{i}\right), \delta\left(x_{j}\right)\right)=\tau^{2} R\left(x_{i}-x_{j}\right)\right)$, the parameters of the model for thrust force are estimated at: $\widehat{\beta_{10}}=1.6062, \widehat{\beta_{11}}=0.8882, \widehat{\beta_{12}}=0.0006$, $\widehat{\beta_{13}}=0.0098$, and $\widehat{\beta_{14}}=0.0019$, These parameters were estimated by Singh et al. [10] using nonlinear regression at $\widehat{\beta_{10}}=1.605, \widehat{\beta_{11}}=0.888, \widehat{\beta_{12}}=0.00058$, $\widehat{\beta_{13}}=0.009$, and $\widehat{\beta_{14}}=0.0018$. For the cutting force, they are estimated at: $\widehat{\beta_{20}}=1.3581, \widehat{\beta_{21}}=0.8888$, $\widehat{\beta_{22}}=0.0014, \widehat{\beta_{23}}=0.0269$, and $\widehat{\beta_{24}}=0.0034$, which were previously estimated at $\beta_{20}=1.358, \beta_{21}=0.888$, $\beta_{22}=0.00139, \beta_{23}=0.0268, \beta_{24}=0.00343$ by Singh et al. [10] using nonlinear regression.

Therefore, the models for the thrust force and the cutting force are calibrated respectively as follows:

$$
\left\{\begin{array}{l}
f_{1}(x)=1.6062 x_{1}{ }^{0.8882} \\
\quad \exp \left\{0.0006 x_{2}-0.0098 x_{3} \exp \left(-0.0019 x_{4}\right)\right\} \\
f_{2}(x)=1.3581 x_{1} 0.8888 \\
\quad \exp \left\{0.0014 x_{2}-0.0269 x_{3} \exp \left(-0.0034 x_{4}\right)\right\} .
\end{array}\right.
$$

It is observed in Figure 2 that the model is fitted excellently and the metamodel is proper. The actual values are shown on the vertical axis and the predicted ones on the parallel axis. Since the dots are near the bisector line, the errors can be negligible. As $\operatorname{cov}\left(\delta\left(x_{i}\right), \delta\left(x_{j}\right)\right)=\tau^{2} R\left(x_{i}-x_{j}\right)$, using Eq. (4), the

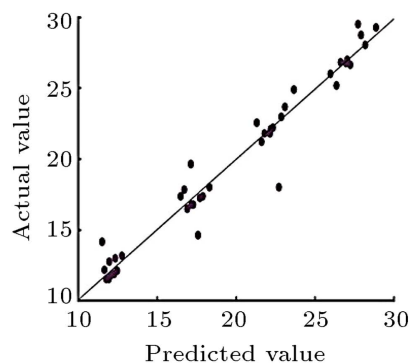

(a)

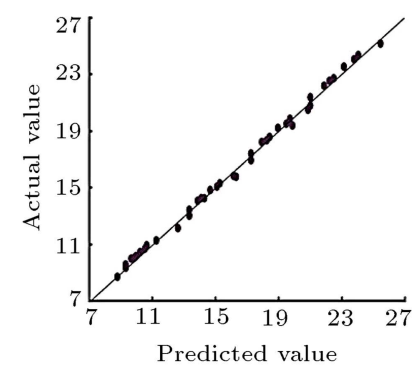

(b)
Figure 2. Actual versus predicted thrust force (a) and cutting force (b) by the engineering model.

covariance between the errors is calculated as:

$$
\operatorname{cov}\left(\varepsilon_{1}, \varepsilon_{2}\right)=\left[\begin{array}{ll}
0.0002 & 0.0001 \\
0.0001 & 0.2825
\end{array}\right] \text {. }
$$

The values of the parameters and variables are listed in Table 4 in order to simplify studying this section.

By applying MANOVA to the engineering model, the factors with the highest level of effects on both variables were detected. This study only reports the results of the Wilk's test, since it is the most commonly used test along with the well-known $\mathrm{F}$ approximation. Compared with $F_{\alpha}$, in which $\alpha=0.01$, all factors are important. The results are reported in Table 5 .

As Roshan and Yan [5] have stated, two-factor interactions are not so imperative, while considering them increases the complexity of the model. Therefore, the related terms are neglected here.

\begin{tabular}{|c|c|c|}
\hline $\begin{array}{c}\text { Parameter/ } \\
\text { variable }\end{array}$ & Description & Value \\
\hline$Y$ & The output variables & $\left(y_{1}, y_{2}\right)$ \\
\hline$q$ & Number of the output variables & 2 \\
\hline$x$ & The vector of input variables & $\left(x_{1} \ldots x_{4}\right)$ \\
\hline$p$ & Number of the input variables & 4 \\
\hline$\eta$ & $\begin{array}{l}\text { The vector of calibration parameters of } \\
\text { the engineering model }\end{array}$ & $\begin{array}{c}(1.605,0.888,0.00058,0.009,0.0018) \\
(1.3581,0.888,0.0014,0.0269,0.0034)\end{array}$ \\
\hline$f(x ; \eta)$ & The engineering model & $\left(f_{1}(x), f_{2}(x)\right)$ \\
\hline$\rho$ & The scale parameter & 1 \\
\hline$\delta(x)$ & The discrepancy function (model bias) & $\left(\delta_{1}, \delta_{2}\right)$ \\
\hline$\varepsilon$ & The random error & $\left(\varepsilon_{1}, \varepsilon_{2}\right)$ \\
\hline$\sigma^{2}$ & The variance of errors & $(0.0118,0.0148)$ \\
\hline$\mu$ & The mean of the discrepancy function & $\left(\mu_{1}, \mu_{2}\right)$ \\
\hline$\tau^{2}$ & The variance of the discrepancy function & $(1.1401,0.3114)$ \\
\hline$R$ & $n \times n$ correlation matrix & $R_{1}, R_{2}$ \\
\hline$\theta$ & The characteristic length scale & $\begin{aligned} \theta_{2}{ }^{\prime} & =(2.6891,21.2868,2.0061,56.5802) \\
\theta_{1}{ }^{\prime} & =(7.774,27.7022,3.1395,265.0827)\end{aligned}$ \\
\hline
\end{tabular}

Table 4. Values of parameters and variables. 
Table 5. Results of general MANOVA applied to the engineering function.

\begin{tabular}{cccccccc}
\hline \multirow{2}{*}{ Factor } & \multirow{2}{*}{$\begin{array}{c}\text { Test } \\
\text { statistic }\end{array}$} & $\boldsymbol{F}$ & \multicolumn{2}{c}{$\boldsymbol{D F}$} & \multirow{P}{*}{$\boldsymbol{F}_{\mathbf{0 . 0 1}}$} & $\begin{array}{c}\text { Being } \\
\text { significant }\end{array}$ \\
\cline { 3 - 5 } & & $\mathbf{N u m}$ & Denom & & & $\sqrt{ }$ \\
$X_{1}$ & 0.00013 & 1133.272 & 6 & 78 & 0.000 & 3.04 & $\sqrt{ }$ \\
$X_{2}$ & 0.40779 & 28.319 & 2 & 39 & 0.000 & 5.19 & $\sqrt{ }$ \\
$X_{3}$ & 0.08162 & 48.757 & 4 & 78 & 0.000 & 3.57 & $\sqrt{ }$ \\
$X_{4}$ & 0.44367 & 24.452 & 2 & 39 & 0.000 & 5.19 & $\sqrt{ }$ \\
\hline
\end{tabular}

Table 6. Results of general MANOVA applied to the discrepancy function.

\begin{tabular}{|c|c|c|c|c|c|c|c|}
\hline \multirow{2}{*}{ Factor } & \multirow{2}{*}{$\begin{array}{c}\text { Test } \\
\text { statistic }\end{array}$} & \multirow{2}{*}{$\boldsymbol{F}$} & \multicolumn{2}{|c|}{$D F$} & \multirow{2}{*}{$P$} & \multirow{2}{*}{$F_{0.01}$} & \multirow{2}{*}{$\begin{array}{c}\text { Being } \\
\text { significant }\end{array}$} \\
\hline & & & Num & Denom & & & \\
\hline$X_{1}$ & 0.78619 & 1.662 & 6 & 78 & 0.142 & 3.04 & $\nabla$ \\
\hline$X_{2}$ & 0.99389 & 0.120 & 2 & 39 & 0.887 & 5.19 & $\otimes$ \\
\hline$X_{3}$ & 0.75552 & 2.934 & 4 & 78 & 0.026 & 3.57 & $\nabla$ \\
\hline$X_{4}$ & 0.99462 & 0.106 & 2 & 39 & 0.900 & 5.19 & $\otimes$ \\
\hline
\end{tabular}

Now, the discrepancy functions should be constructed. By fitting a GP, the parameters of the discrepancy function will be calculated by Eqs. (3) and (4):

For the thrust force:

$$
\begin{aligned}
\sigma^{2} & =0.0118, \quad \tau^{2}=1.1401, \\
\theta^{\prime} & =(2.6891,21.2868,2.0061,56.5802),
\end{aligned}
$$

For the cutting force:

$$
\begin{aligned}
& \sigma^{2}=0.0148, \quad \tau^{2}=0.3114 \\
& \theta^{\prime}=(7.774,27.7022,3.1395,265.0827) .
\end{aligned}
$$

By applying MANOVA to the discrepancy function, the most effective factors on both variables are detected. The results of the Wilk's test are reported in Table 6. As observed, compared with $F_{0.01}$, all factors are insignificant. All of the factors are effective on the engineering models and none of them are effective on the discrepancy functions. This ineffectiveness means that the responses $(y)$ have been modeled as much as possible and the remaining, which are not modeled, are part of $y$, which is the random error, indeed.

The main effects of $f(x)$ and $f(x)+\delta(x)$ are presented by Figure 3. It is seen that they are so close and almost covered by each other. Hence, the discrepancy function is not impressive and it can be neglected. However, here, the model adjustment approach is continued.

The following adjusted model seems to be physically meaningful for both forces:

$$
\hat{g}(x ; \gamma)=\hat{f}\left(x_{1}, x_{2}, x_{3}, x_{4}\right) .
$$

Therefore, according to Eq. (19), the engineeringstatistical models for the thrust force and the cutting force are adjusted, respectively.
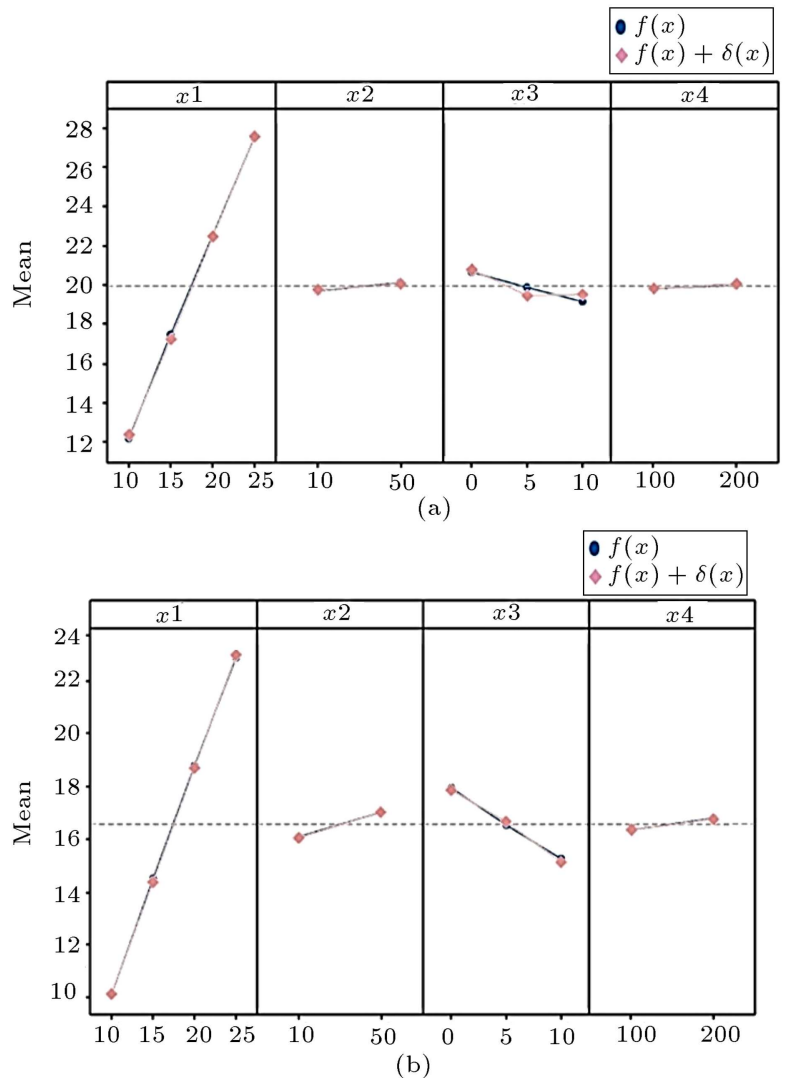

Figure 3. The main effects of $f(x)$ and $f(x)+\delta(x)$ on (a) Thrust force and (b) cutting force.

$$
\left\{\begin{array}{l}
g(x)=1.6062 x_{1}^{0.8882} \\
\quad \exp \left\{0.0006 x_{2}-0.0098 x_{3} e^{-0.0019 x_{4}}\right\} \\
g(x)=1.3581 x_{1}^{0.8888} \\
\quad \exp \left\{0.0014 x_{2}-0.0269 x_{3} e^{-0.0034 x_{4}}\right\}
\end{array}\right.
$$

Considering $\hat{y}=\hat{f}+\hat{\delta}$ for the thrust force, they are estimated at: $\hat{f}=19.9315, \hat{\delta}=0.0091, \hat{y}=19.9315+$ 
$0.0091=19.9406$ and for the cutting force, they are estimated at: $\hat{f}=16.5787, \hat{\delta}=0.0031, \widehat{y}=16.5787+$ $0.0031=16.5819$. Apparently, for the thrust force, the mean remaining error is 0.0091 and for the cutting force, the value is 0.0032 .

The Mean Squared Prediction Error (MSPE) seems to be a proper index for quantifying the improvement:

$$
M S P E=\frac{1}{n} \sum_{i=1}^{n}\left[y_{i}-f\left(x_{i}\right)\right]^{2} .
$$

For the thrust force, the index was obtained 1.48 by Kennedy and O'Hagan adjustment model [2], 2.47 by the adjustment model presented by Roshan and Yan [5], and $1.9425 \times 10^{-4}$ by the currently proposed model. For the cutting force, the index was obtained 0.21 by the Kennedy and O'Hagan [2], 1.41 by the Roshan and Yan [5], and $1.6 \times 10^{-8}$ by the presented model.

As the above analysis shows, the errors of the proposed method are fairly near to zero. The reason is that the initial experiments are used again. Therefore, to validate the methodology, cross-validation and a second approach are applied. These approaches are stated in the next section.

\subsection{Validation}

As explained before, validation is needed to ensure that the methodology works fine. Hence, to validate the methodology, the cross-validation approach is applied. In order to accomplish the cross-validation, the programming should be repeated 48 times. In each run, one observation should be omitted. Calibrating and adjusting the model for the remaining 47 observations, the results are applied to the new experiment (omitted one). Calculating the MSPE for the entire experiments, the average error squares are calculated for both the thrust force (Eq. (22)) and the cutting force (Eq. (23)):

$$
\begin{aligned}
& M S P E=\frac{1}{n}\left(\sum_{i=1}^{n}\left[\hat{y}_{1, i}-y_{1, i}\right]^{2}\right)=4.0691, \\
& M S P E=\frac{1}{n}\left(\sum_{i=1}^{n}\left[\hat{y}_{2, i}-y_{2, i}\right]^{2}\right)=1.5378 .
\end{aligned}
$$

It is seen that the methodology gets solutions fairly close to those of the previous works and it can be considered valid now.

Again, as a second approach for validation of the methodology, new experiments should be conducted. To do new experiments, a normal distribution with mean $\bar{x}$ and variance $\operatorname{cov}(x)$ is used to create 48 new random sets of input variables. The same procedure is done for output variables. That is, the previous outputs and the covariance matrix between them are set for the outputs, and for the inputs Eq. (24) is used:

$$
\begin{aligned}
\mu_{x} & =\left[\begin{array}{llll}
20 & 30 & 5 & 22.5
\end{array}\right] ; \\
\sigma_{x} & =\left[\begin{array}{cccc}
16.82 & 0 & 0 & 0 \\
0 & 403.740 & 0 & \\
0 & 0 & 16.82 & 0 \\
0 & 0 & 0 & 56.77
\end{array}\right] .
\end{aligned}
$$

By applying the parameters discrepancy function, estimated previously, the error is calculated for both outputs by Eq. (25):

$$
\begin{aligned}
M S P E & =\frac{1}{n}\left(\sum_{i=1}^{n}\left[\hat{y}_{1, i}-y_{1, i}\right]^{2}+\sum_{i=1}^{n}\left[\hat{y}_{2, i}-y_{2, i}\right]^{2}\right) \\
& =0.0014 .
\end{aligned}
$$

Again, not using the discrepancy function, the errors are calculated by Eq. (26):

$$
\begin{aligned}
M S P E & =\frac{1}{n}\left(\sum_{i=1}^{n}\left[f_{1, i}-y_{1, i}\right]^{2}+\sum_{i=1}^{n}\left[f_{2, i}-y_{2, i}\right]^{2}\right) \\
& =170.5232 .
\end{aligned}
$$

It is seen that even for the created variables, the discrepancy function is proper. Thus, the methodology works better than the previous one, as validated by the new inputs and outputs.

\section{Conclusion}

In this paper, by integrating the engineering and statistical approaches, a novel engineering-statistical approach was introduced for calibrating and adjusting a model for complex systems. The methodology was applied to the Laser-Assisted Micro Machining (LAMM) problem in order to study the thrust force and cutting force as the outputs, simultaneously, for the first time. Considering Mean Squared Prediction Error (MSPE) as the index of model adequacy, the values were obtained $1.9425 \times 10^{-4}$ for thrust force and $1.6 \times 10^{-8}$ for cutting force by the presented model. The proposed model worked better by the index than the models introduced previously. The errors were considerably reduced because the data from which the model was developed and the data to which the model was applied were the same. Therefore, to validate the methodology, the proposed approach was applied to new data and MSPE was gained 0.0014, which was less than when the discrepancy function was not applied (170.52) yet. Cross-validation was applied to all the experiments as well. For the thrust force and cutting force, MSPE was calculated 4.0691 and 1.5378 , respectively. In this paper, we focused on presenting a methodology for calibration and adjustment of a multi-output model. On the other 
hand, the experimental designs played a crucial role in both computer experiments and physical experiments. For further research work in future, using the spacefilling design for the computer design can be a new direction. Even the orthogonal space-filling design may be studied to obtain more efficient estimations.

\section{References}

1. Singh, R.K. and Melkote, S.N. "Force modeling in laser assisted micro-grooving including the effect of machine deflection", ASME Journal of Manufacturing Science and Engineering, 131(1), pp. 1-9 (2009).

2. Kennedy, M.C. and O'Hagan, A. "Bayesian calibration of computer models", Journal of Royal Statistical Society - Series B, 63, pp. 425-464 (2001).

3. Roshan, V.J. and Melkote, S.N., Statistical Adjustments to Engineering Models, Georgia Institute of Technology, pp. 1-24 (2008).

4. Yan, H. "Statistical adjustment, calibration, and uncertainty quantification of complex computer models", Ph.D. Thesis, H. Milton Stewart School of Industrial and Systems Engineering, Georgia Institute of Technology (2014).

5. Roshan, V.J. and Yan, H. "Engineering-driven statistical adjustment and calibration", Technometrics, 57(2), pp. 257-267 (2015).

6. Sheikhi, H. and Saghaie, A. "Developing an engineering-statistical model for estimating aerodynamic coefficients of helicopter fuselage", Chinese Journal of Aeronautics, 30(1), pp. 175-185 (2017).

7. Rahimi, M., Shafieezadeh, A., Wood, D., et al. "Bayesian calibration of multi-response systems via multivariate Kriging: Methodology and geological and geotechnical case studies", Engineering Geology, 260, 105248, ISSN 0013-7952 (2019). https://doi.org/10.1016/j.enggeo.2019.105248

8. Daniel, W., David, M., James, W.J., and François Working with Dynamic Crop Models Methods, Tools and Examples for Agriculture and Environment, Elsevier, 3rd Edition (2019).

9. Yan, G., Sun, H., and Waisman, H. "A guided Bayesian inference approach for detection of multiple flaws in structures using the extended finite element method", Computers and Structures, 152, pp. 27-44 (2015).

10. Singh, R.K., Josef, V.R., and Melkote, S.N. "A statistical approach to the optimization of a laserassisted micromachining process", International Journal of Advanced Manufacturing Technology, 53, pp. 221-230 (2011).

11. Allaire, G., Numerical Analysis and Optimization: An Introduction to Mathematical Modelling and Numerical Simulation, Oxford, New York (2007).

12. Nocedal, J. and Wright, S.J., Numerical optimization, Springer, New York (2006).
13. Szabó, B.A. "The use of a priori estimates in engineering computations", Computer Methods in Applied Mechanics and Engineering, 82(1-3), pp. 139-154 (1990).

14. Mathelin, L. and Hussaini, M.Y., A Stochastic Collocation Algorithm for Uncertainty Analysis, NASA Center for AeroSpace Information (CASI), pp. 1-16 (2003).

15. Vepsä, A., Haapaniemi, H., Luukkanen, P., et al. "Application of finite element model updating to a feed water pipeline of a nuclear power plant", Nuclear Engineering and Design, 235(17-19), pp. 1849-1865 (2005).

16. Palumbo, G., Piccininni, A., Piglionico, V., et al. "Modelling residual stresses in sand-cast superduplex stainless steel", Journal of Materials Processing Technology, 217, pp. 253-261 (2015).

17. Stickler, B. and Schachinger, E., Basic Concepts in Computational Physics, Springer, New York (2014).

18. Sena, P.K. and Silvapulle, M.J. "An appraisal of some aspects of statistical inference under inequality constraints", Journal of Statistical Planning and Inference, 107, pp. 3-43 (2002).

19. Kumar, R., Tewari, P.C., and Khanduja, D. "Parameters optimization of fabric finishing system of a textile industry using teaching-learning-based optimization algorithm", International Journal of Industrial Engineering Computations, 6(2), pp. 221-234 (2018).

20. Roussas, G., An Introduction to Measure-Theoretic Probability, 2nd Ed., Elsevier, USA (2014).

21. Zio, E., The Monte Carlo Simulation Method for System Reliability and Risk Analysis, Springer, London (2013).

22. Dong, L., Xiaojing, L., and Yanhua, Y. "Investigation of uncertainty quantification method for be models using MCMC approach and application to assessment with feba data", Annals of Nuclear Energy, 107, pp. 62-70 (2017).

23. Parnianifard, A., Azfanizam, A.S., Ariffin, M.K.A., et al. "An overview on robust design hybrid metamodeling: Advanced methodology in process optimization under uncertainty", International Journal of Industrial Engineering Computations, 9(1), pp. 1-32 (2018).

24. Park, I. and Grandhi, R.V. "A Bayesian statistical method for quantifying model form uncertainty and two model combination methods", Reliability Engineering and System Safety, 129, pp. 46-56 (2014).

25. Caiado, C.C.S. and Goldstein, M. "Bayesian uncertainty analysis for complex physical systems modelled by computer simulators with applications to tipping points", Communications in Nonlinear Science and Numerical Simulation, 26(1-3), pp. 123-136 (2015).

26. Wong, S.W.K., Lum, C., Wu, L., et al. "Quantifying uncertainty in lumber grading and strength prediction: a Bayesian approach", Technometrics, 58(2), pp. 236243 (2016). 
27. Sankararaman, S. and Mahadevan, S. "Integration of model verification, validation, and calibration for uncertainty quantification in engineering systems", Reliability Engineering and System Safety, 138, pp. 194-209 (2015).

28. Duru, O., Bulut, E., and Yoshida, Sh. "A fuzzy extended delphi method for adjustment of statistical time series prediction: an empirical study on dry bulk freight market case", Expert Systems with Applications, 39(1), pp. 840-848 (2012).

29. Azzimonti, D., Bect, J., Chevalier, C., et al., Quantifying Uncertainties on Excursion Sets under a Gaussian Random Field Prior, Cornell University (arXiv), (2015).

30. Reid, N. "Statistical sufficiency", International Encyclopedia of the Social \& Behavioral Sciences, 2, pp. 418-422 (2015).

31. Junaid, M.M. and Wani, M.F. "Modelling and analysis of tool wear and surface roughness in hard turning of AISI D2 steel using response surface methodology", International Journal of Industrial Engineering Computations, 9(1), pp. 63-74 (2018).

32. Brandt, S., Data Analysis: Statistical and Computational Methods for Scientists and Engineers, Springer, 4, New York (2014).

33. Korunović, N., Madić, M., Trajanović, M., and Radovanović, M. "A procedure for multi-objective optimization of tire design parameters", International Journal of Industrial Engineering Computations, 6(2), pp. 199-210 (2015).

34. Lin, H.D. and Lin, W.T. "Automated process adjustments of chip cutting operations using neural network and statistical approaches", Expert Systems with Applications, 36, pp. 4338-4345 (2009).

35. Lunardon, N. and Ronchetti, E. "Composite likelihood inference by nonparametric saddle point tests", Computational Statistics and Data Analysis, 79, pp. 80-90 (2014).

36. Pratola, M.T. and Higdon, D.M. "Bayesian additive regression tree calibration of complex high-dimensional computer models", Technometrics, 58(2), pp. 166-179 (2016).

37. Recep, M.G., Seung-Kyum, C., and Christopher, J.S. "Uncertainty quantification and validation of $3 \mathrm{D}$ lattice scaffolds for computer-aided biomedical applications", Journal of the Mechanical Behavior of Biomedical Materials, 71, pp. 428-440 (2017).

38. Saikumar, R.Y., Michael, G.G., Christos, A., et al. "Bayesian uncertainty quantification and propagation for validation of a microstructure sensitive model for prediction of fatigue crack initiation", Reliability Engineering \& System Safety, 164, pp. 110-123 (2017).

39. Chen, R.B., Wang, W., and Wu, C.F.J. "Sequential designs based on bayesian uncertainty quantification in sparse representation surrogate modeling", Technometrics, 59(2), pp. 139-152 (2017).

40. Neal, R.M. "Regression and classification using gaussian process priors", Bayesian Statistics, 6, pp. 475501 (1998).

41. Mondal, A., Mallick, B., Efendiev, Y., and DattaGupta, A. "Bayesian uncertainty quantification for subsurface inversion using a multiscale hierarchical model", Technometrics, 56(3), pp. 381-392 (2014).

42. Díaz-García, J.A. "On generalized multivariate analysis of variance", Brazilian Journal of Probability and Statistics, 25(1), pp. 1-13 (2011).

\section{Biographies}

Zeinab Khalaj is currently a $\mathrm{PhD}$ candidate in Industrial Engineering at K.N. Toosi University of Technology, Tehran, Iran, and simultaneously a lecturer at Islamic Azad University, Karaj, Iran. Her main areas of interest are statistical analysis and uncertainty quantification as well as project management.

Abdollah Aghaie is a Professor of Industrial Engineering at K.N. Toosi University of Technology in Tehran, Iran. He received his BSc from Sharif University of Technology in Tehran, MSc from New South Wales University in Sydney, Australia, and PhD from Loughborough University in the UK. His main research interests are in modeling and simulation, queuing System, quality management and control, supply chain, and data science.

Yaser Samimi is a faculty member of the Industrial Engineering Department at K.N. Toosi Unievrsity of Technology, Tehran, Iran. He received his $\mathrm{PhD}$ in Industrial Engineering from the same university and holds both BSc and MSc in Industrial Engineering. His primary research interests include statistical pattern recognition, statistical process control, time series analysis, and change point detection methods. He is a member of the Iranian Statistical Association and Iran Institute of Industrial Engineering. 